\title{
Point-of-care transthoracic echocardiography as an alternative to transesophageal echocardiography to confirm internal jugular guidewire position
}

\author{
Ramiro Arellano, MD • Aliya Nurmohamed • \\ Amir Rumman, BSc $\cdot$ Brian Milne, MD • \\ Robert Tanzola, MD
}

Received: 15 September 2011/ Accepted: 6 October 2011 / Published online: 28 October 2011

(C) Canadian Anesthesiologists' Society 2011

Unintentional needle puncture of the carotid artery (CA) during internal jugular vein (IJV) cannulation occurs relatively frequently. ${ }^{1}$ Serious sequelae (e.g., hematoma, airway compromise, stroke, and death) usually occur when this error is not detected, and the CA is subsequently dilated with the large-bore introducer sheath. Methods employed to detect CA puncture include blood colour and flow characteristics, manometry, and pressure transduction of the needle. Ultrasonographic visualization of the IJV and CA prior to cannulation is endorsed as a method to improve safety. ${ }^{2}$ However, this technology does not completely eliminate accidental CA injury. Ultrasonography of the neck visualizes the guidewire for only a short distance, leaving room for posterior vein wall penetration outside the field of view.

In many centres providing cardiac anesthesia, transesophageal echocardiography (TEE) is used to visualize the guidewire in the superior vena cava (SVC) or right atrium (RA) before the introducer sheath is inserted. ${ }^{3}$ The guidewire, seen in the bicaval view, confirms appropriate wire placement.
Point-of-care ultrasonography is used increasingly by anesthesiologists, intensivists, and emergency medicine specialists to diagnose and treat hemodynamically unstable patients. Transthoracic echocardiographic (TTE) apical and subcostal four-chamber views are routinely obtained during these point-of-care assessments. We now use these TTE views to confirm appropriate IJV puncture and guidewire advancement in venous structures. During the initial venous puncture, blood is aspirated into a syringe and reinjected quickly. This produces microbubbles that are seen entering the RA, thereby confirming venous puncture (Fig. 1, Clip 1). The advanced guidewire is visualized in the SVC, RA, or inferior vena cava. In our experience, both the subcostal (Fig. 2, Clip 2) and subcostal bicaval (Fig. 3, Clip 3) views provide optimal sonographic windows.

Guidewire confirmation with point-of-care TTE during IJV cannulation provides an alternative to TEE for confirming appropriate guidewire placement. Also, it may aid in reducing the incidence of CA injury associated with IJV cannulation.
Electronic supplementary material The online version of this article (doi:10.1007/s12630-011-9608-1) contains supplementary material, which is available to authorized users.

R. Arellano, MD $(\bowtie) \cdot$ A. Nurmohamed · A. Rumman, BSc .

B. Milne, MD · R. Tanzola, MD

Queen's University, Kingston, ON, Canada

e-mail: arellanr@kgh.kari.net 

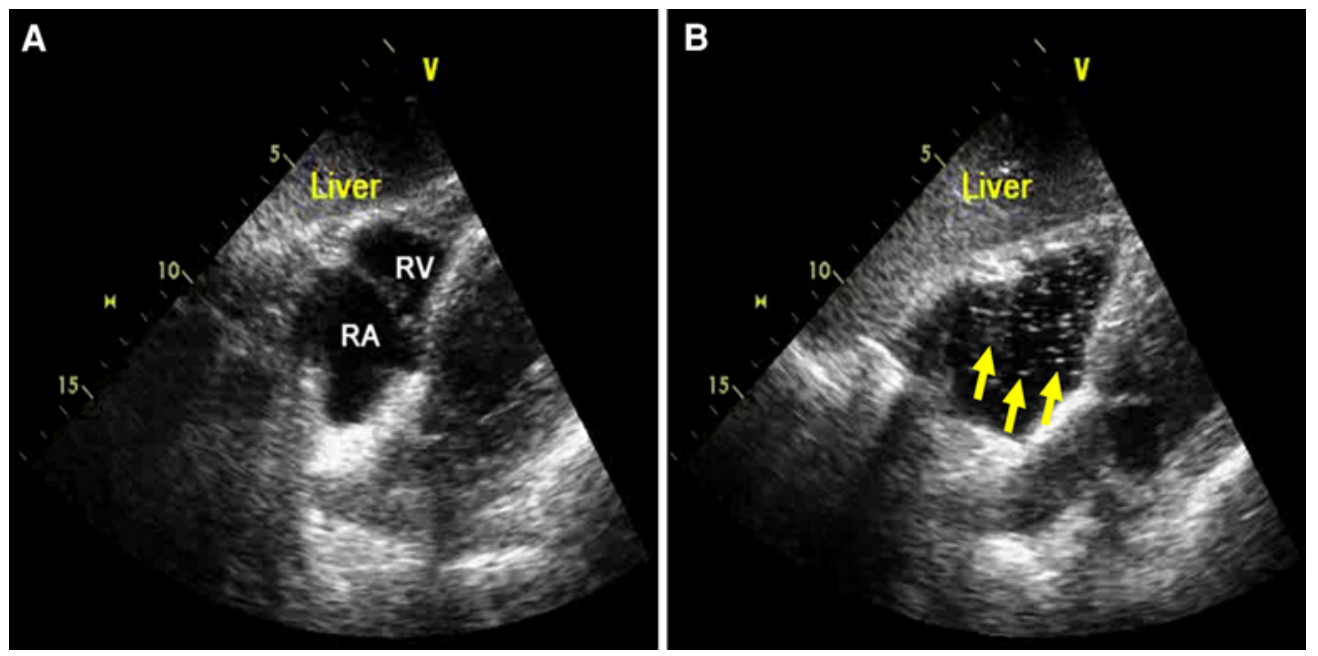

Fig. 1 Preoperative transthoracic echocardiography shows the right atrium (RA) and right ventricle (RV) in subcostal view before (A) and after (B) aspiration and reinjection of $5 \mathrm{~mL}$ of blood through the catheter. The arrowheads denote microbubbles and the resulting opacification of the RA $+\mathrm{RV}$
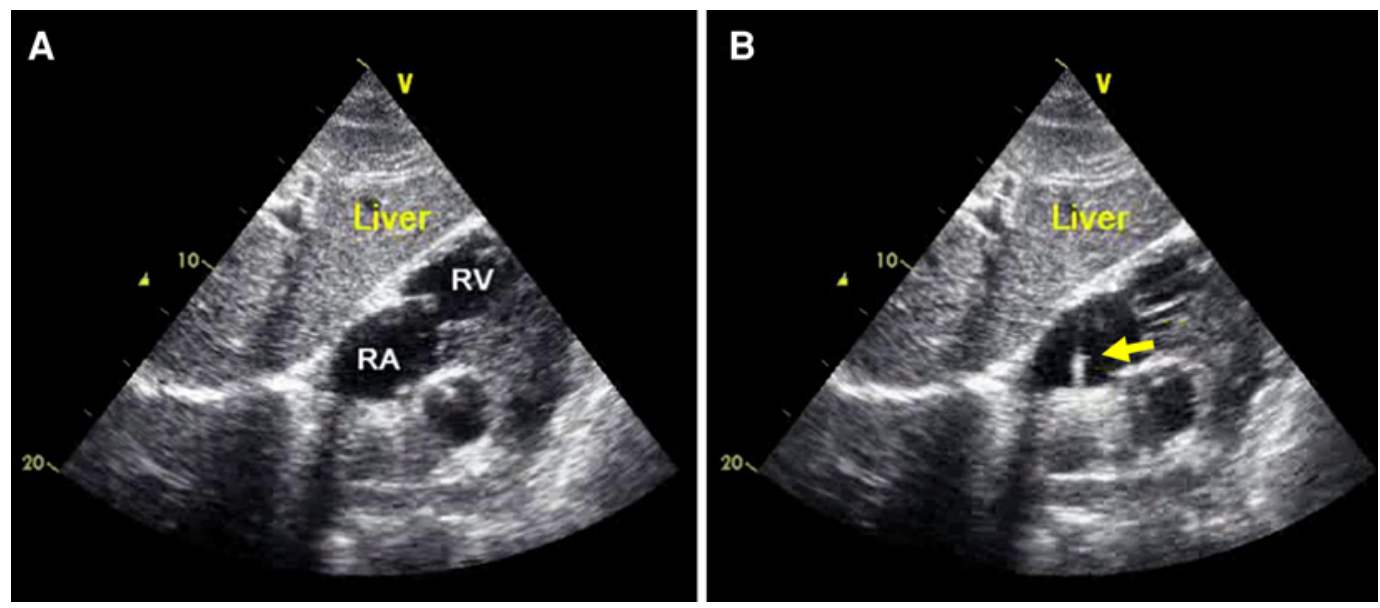

Fig. 2 Preoperative transthoracic echocardiography, subcostal view, shows the right ventricle (RV) and right atrium (RA) before (A) and after (B) guidewire insertion into the RA

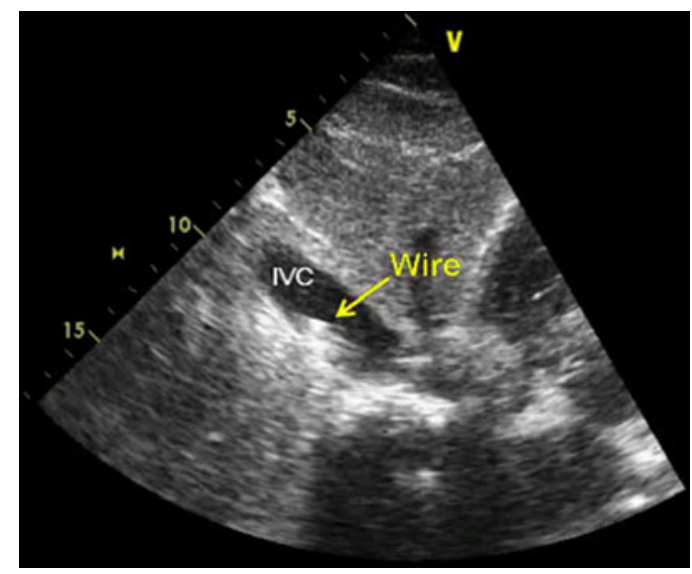

Fig. 3 Preoperative transthoracic echocardiography, subcostal bicaval view, shows the tip of the guidewire in the inferior vena cava (IVC)
Conflicts of interest None declared.

\section{References}

1. McGee DC, Gould MK. Preventing complications of central venous catheterization. N Engl J Med 2003; 348: 1123-33.

2. Blaivas $M$, Adhikari $S$. An unseen danger: frequency of posterior vessel wall penetration by needles during attempts to place internal jugular vein central catheters using ultrasound guidance. Crit Care Med 2009; 37: 2345-9.

3. Sawchuk C, Fayad A. Confirmation of internal jugular guide wire position utilizing transesophageal echocardiography. Can J Anesth 2001; 48: 688-90. 